GEOMETRY IN NONLINEAR CONTROL

AND DIFFERENTIAL INCLUSIONS

BANACH CENTER PUBLICATIONS, VOLUME 32

INSTITUTE OF MATHEMATICS

POLISH ACADEMY OF SCIENCES

WARSZAWA 1995

\title{
TOWARD A NOTION OF CANONICAL FORM FOR NONLINEAR SYSTEMS
}

\author{
G. CONTE \\ Dipartimento di Automatica ed Elettronica, Università di Ancona \\ Via Brecce Bianche, 60131 Ancona, Italy \\ A. M. PERDON \\ Dipartimento di Matematica "V. Volterra", Università di Ancona \\ Via Brecce Bianche, 60131 Ancona, Italy \\ C. H. $\mathrm{MOOG}$ \\ Lab. Automatique de Nantes, U.A. CNRS, ECN/Univ. de Nantes \\ 1 rue de la Nö̈, Nantes, France
}

1. Introduction. In this expository paper we summarize the results of [23], [1] and [20] about the construction of a canonical form for nonlinear systems similar to the Morse canonical form for linear systems.

The interest in canonical forms with respect to groups of transformations is due to the fact that they provide useful tools for analizing the dynamical properties of systems and for studying the solvability of control problems. In the linear framework, the Morse canonical form displays the properties which are invariant with respect to the transformations commonly used for modifying the dynamics or for designing observers ([21], [16]), namely static state-feedbacks and output injections, together with changes of basis.

In a nonlinear framework, canonical forms, in a broad sense, have been considered from different point of view by many authors ([28], [14], [6], [7], [8], [19], [17]). At the present stage of the research, the class of nonlinear transformation that appears interesting to take into account contains feedbacks and change of coordinates that involve the derivatives of the input and, similarly, output in-

1991 Mathematics Subject Classification: 93B10, 93C10, 93B17, 93B25.

This work was performed with the financial support of NATO.

The paper is in final form and no version of it will be published elsewhere. 
jections that involve the derivatives of the output. From this point of view, the picture is reasonably clear and well understood for what concerns the notion of feedback and change of coordinates, and it has been satisfactorily formalised by means of the notion of quasi-static feedback in [2].

Results on the construction of a canonical form with respect to generalized feedbacks and changes of coordinates involving the derivatives of the inputs were obtained in [23]. After introducing, in Section 2, some preliminary notions, we discuss these results in detail in Section 3. Essentially, they show that it is possible, using a particular quasi-static feedback, to decompose a given affine nonlinear system into three subsystems, two of which correspond to suitable subsystems in the decomposition induced, in the linear case, by the Morse canonical form. In doing so, the nonlinear system is made maximally unobservable with respect to the considered class of transformations and then, by means of a very particular additive output injection, it can be linearized from an input/output point of view. The decomposition displays the observable and controllable part and the observable and noncontrollable part of the transformed system, as well as two lists of invariants, corresponding respectively to the structure at infinity and to the Morse list $I_{3}$ (that is a part of the observability indices, after factoring out the maximum controlled invariant subspace in the kernel of the output map) in the linear case.

After [23], further work has been carried on in [1] and [20] in order to obtain a complete analogy with the linear situation, in which, by means of output injection, one succeeds in decoupling the nonobservable subsystem and then in splitting it into a controllable and a noncontrollable part. Actually, the situation appear more difficult in the nonlinear case, mainly because a suitable generalization of the notion of output injection is not easy to be found.

In Section 5 we describe the results obtained in that direction. In particular, we first propose a definition of generalized output injection, which is consistent with the definition of quasi-static feedback, and which behaves well with respect to the observability. Then, we show that the nonobservable subsystem can be decoupled by means of a generalized output injection when a suitable condition holds. In such case one obtains a system representation that is close to that obtained by the Morse canonical form in the linear case.

2. Preliminaries. In order to clarify our objective, we briefly recall in this Section the properties of the Morse canonical form for linear systems. Then we will introduce, in the spirit of [3], some differential algebraic tools that will be useful for dealing with the nonlinear systems we are going to consider.

2.1. The Morse canonical form for linear systems. Given a linear system $\Sigma$ represented by a set of equations of the form

$$
\left\{\begin{array}{l}
\dot{x}(t)=A x(t)+B u(t) \\
y(t)=C x(t)
\end{array}\right.
$$


where $x \in X=\Re^{n}, u \in U=\Re^{m}, y \in Y=\Re^{p}$, and $A, B, C$ are real matrices of suitable dimensions, one is mainly interested in considering the following class of trasformations:

- changes of basis in the vector spaces $X, U, Y$;

- static state feedbacks of the form $u=F x+G v$, where $F$ and $G$ are real matrices of suitable dimensions and $G$ is invertible;

- output injections, that modify the system dynamics by the assignment $\dot{x} \mapsto$ $\dot{x}+K y$, where $K$ is a real matrix of suitable dimensions.

More formally, one considers the group of all transformations acting on the set of triples $(A, B, C)$ in the following way:

$$
(A, B, C) \longmapsto\left(T(A+B F+K C) T^{-1}, T B G, H C T^{-1}\right)
$$

with $G$ and $H$ invertible.

Assuming that $\operatorname{rank} B=m$ and $\operatorname{rank} C=p$, the invariants with respect to the action of such group of transformations are displayed by the Morse canonical form of the triple $(A, B, C)([21])$. Without entering in the details, let us recall that associating to $\Sigma$ the system matrix

$$
\left(\begin{array}{cc}
z I-A & B \\
C & 0
\end{array}\right)
$$

we obtain, for a triple in canonical form, a system matrix having the following structure

$$
\left(\begin{array}{cccccc}
z I-A_{1} & 0 & 0 & 0 & B_{1} & 0 \\
0 & z I-A_{2} & 0 & 0 & 0 & B_{2} \\
0 & 0 & z I-A_{3} & 0 & 0 & 0 \\
0 & 0 & 0 & z I-A_{4} & 0 & 0 \\
C_{1} & 0 & 0 & 0 & 0 & 0 \\
0 & 0 & C_{3} & 0 & 0 & 0
\end{array}\right)
$$

where

- $A_{1}, A_{2}$ and $A_{3}$ are in Jordan form,

- $B_{1}$ and $B_{2}$ are block diagonal matrices, with blocks of the form $\left(\begin{array}{c}0 \\ 0 \\ \vdots \\ 0 \\ 1\end{array}\right)$,

- $C_{1}$ and $C_{3}$ are block diagonal matrices, with blocks of the form $\left(\begin{array}{llll}1 & 0 & \ldots & 0\end{array}\right)$.

The block decomposition of the system matrix corresponds to an underlying decomposition of the state space of the form $X=X_{1} \oplus X_{2} \oplus X_{3} \oplus X_{4}$ where

- $X_{2}:=\mathcal{R}^{\star}$ is the maximum controllability subspace in $\operatorname{Ker} C$;

- $X_{2} \oplus X_{4}:=\mathcal{V}^{\star}$ is the maximum controlled invariant subspace in $\operatorname{Ker} C$; 

$\operatorname{Im} B$.

- $X_{1} \bigoplus X_{2}:=\mathcal{S}^{\star}$ is the minimum conditionally invariant subspace containing

The reduction of a given system matrix to the Morse canonical form is accomplished by applying suitable change of basis, a feedback that makes the system maximally unobservable, and a suitable output injection that makes the system maximally uncontrollable [21], [16]).

The invariants of the triple $(A, B, C)$ displayed by the Morse canonical form are given by:

- the list $I_{1}=\left\{n_{1}, \ldots, n_{k}\right\}$ of the controllability indices of the pair $\left(A_{1}, B_{1}\right)$ (these coincide also with the observability indices of the pair $\left(A_{1}, C_{1}\right)$ and with the structure at $\infty$ of $\Sigma$ ),

- the list $I_{2}$ of the controllability indices of the pair $\left(A_{2}, B_{2}\right)$,

- the list $I_{3}$ of the observability indices of the pair $\left(A_{3}, C_{3}\right)$,

- the list $I_{4}=\left\{p_{1}(z), \ldots, p_{r}(z)\right\}$ of the invariant polynomials of $A_{4}$, or the transmission polynomials of $\Sigma$

2.2. Algebraic-differential tools for nonlinear systems. Consider a nonlinear control system $\Sigma$ of the form:

$$
\Sigma=\left\{\begin{array}{l}
\dot{x}=f(x)+g(x) u \\
y=h(x)
\end{array}\right.
$$

where $x \in \Re^{n}, u \in \Re^{m}, y \in \Re^{p}$, and where the components of $f(),. g($.$) and h($. are meromorphic functions of the variable $x$ on an open subset $\mathcal{D} \subseteq \Re^{n}$. In order to associate with $\Sigma$ a vector space of formal differentials we need to carry on, in the line of [3], the following construction.

Let $\mathcal{C}$ denote the infinite set of real indeterminates defined by

$$
\mathcal{C}=\left\{x_{i}, i=1, \ldots, n ; u_{j}^{(k)}, j=1, \ldots, m, k \geq 0\right\} .
$$

For any positive integer $r$, we use the first $r$ elements of $\mathcal{C}$ for denoting the coordinates of a point in $\Re^{r}$. Hence, a function from $\Re^{r}$ to $\Re$ will be written as a function in the first $r$ indeterminates of $\mathcal{C}$.

The set $\mathcal{K}_{r}$, consisting of all meromorphic functions from $\Re^{r}$ to $\Re$ is naturally endowed with a differential field structure determined by the usual partial derivative operators $\partial / \partial x_{i}$ and $\partial / \partial u_{j}^{(k)}$. The union $\bigcup_{r} \mathcal{K}_{r}$ will be denoted simply by $\mathcal{K}$. Any element of $\mathcal{K}$ is a meromorphic function depending on a finite subset of indeterminates of $\mathcal{C}$ and it will be simply denoted by $F\left(\left\{x_{i}, u_{j}^{(k)}\right\}\right)$. Quite obviously, $\mathcal{K}$ has a field structure, moreover it can be endowed with a differential structure determined by the system $\Sigma$. To this end, let us define a derivative operator $\delta$, acting on $\mathcal{K}$ as follows:

- $\delta x_{i}=f_{i}(x)+g_{i}(x) u^{(0)}$ for all $i=1, \ldots, n$,

- $\delta u_{j}^{(k)}=u_{j}^{(k+1)}$ for $\mathrm{k} \geq 0$ and for all $j=1, \ldots, m$,

- $\delta F\left(\left\{x_{i}, u_{j}^{(k)}\right\}\right)=\sum_{i=1}^{n}\left(\partial F / \partial x_{i}\right) \delta x_{i}+\sum_{j=1, \ldots, m ; k \geq 0}\left(\partial F / \partial u_{j}^{(k)}\right) \delta u_{j}^{(k)}$. 
We can consider now abstract vector spaces spanned over the differential field $\mathcal{K}$. In particular, denoting by $d \mathcal{C}$ the infinite set of symbols

$$
d \mathcal{C}=\left\{d x_{i}, i=1, \ldots, n ; d u_{j}^{(k)}, j=1, \ldots, m, k \geq 0\right\}
$$

we can introduce the vector space $\mathcal{E}$, spanned over $\mathcal{K}$ by the elements of $d \mathcal{C}$, and we write $\mathcal{E}=\operatorname{span} d \mathcal{C}$. Any element in $\mathcal{E}$ is a vector of the form

$$
v=\sum_{i=1}^{n} F_{i} d x_{i}+\sum_{j=1, \ldots, m ; k \geq 0} F_{j k} d u_{j}^{(k)}
$$

where only a finite number of coefficients $F_{i}, F_{i j}$ are nonzero elements of $\mathcal{K}$.

We define now an operator from $\mathcal{K}$ to $\mathcal{E}$, that by abuse of notation will be denoted by $d$, in the following way :

$$
d F\left(\left\{x_{i}, u_{j}^{(k)}\right\}\right)=\sum_{i=1}^{n}\left(\partial F / \partial x_{i}\right) d x_{i}+\sum_{j=1, \ldots, m ; k \geq 0}\left(\partial F / \partial u_{j}^{(k)}\right) d u_{j}^{(k)} .
$$

The elements of $\mathcal{E}$ will be called one-forms and we will say that $v \in \mathcal{E}$ is an exact one-form if $v=d F$ for some $F \in \mathcal{K}$. We will usually refer to $d F$ as to the differential of $F$.

The vector space $\mathcal{E}$ may be used for investigating the structural properties of $\Sigma$. By remarking that the components of the output $y$ and their time derivatives

$$
\begin{aligned}
\dot{y} & =\dot{y}(x, u)=\frac{\partial y}{\partial x}[f(x)+g(x) u], \\
& \vdots \\
y^{(k+1)} & =y^{(k+1)}\left(x, u, \ldots, u^{(k)}\right)=\frac{\partial y^{(k)}}{\partial x}[f(x)+g(x) u]+\sum_{i=0}^{k-1} \frac{\partial y^{(k)}}{\partial u^{i}} u^{(i+1)}
\end{aligned}
$$

are meromorphic functions of $x, u, \ldots, u^{(k)}$, and hence elements of $\mathcal{K}$, we get,by differentiating, a vector in $\mathcal{E}$ for each component $y_{i}^{(k)}$ of $y^{(k)}$ and we set $d y^{(k)}=$ $\left\{d y_{i}^{(k)}, i=1, \ldots, p\right\}$. The chain of subspaces $\mathcal{E}_{0} \subset \mathcal{E}_{1} \subset \ldots \subset \mathcal{E}_{n}$ of $\mathcal{E}$ defined by

$$
\begin{aligned}
\mathcal{E}_{0} & =\operatorname{span}\{d x\}, \\
\mathcal{E}_{1} & =\operatorname{span}\{d x, d \dot{y}\}, \\
& \vdots \\
\mathcal{E}_{n} & =\operatorname{span}\left\{d x, d \dot{y}, \ldots, d y^{(n)}\right\}
\end{aligned}
$$

is naturally associated to the system $\Sigma$.

Definition 1. Given the chain of vector spaces $\mathcal{E}_{0} \subset \mathcal{E}_{1} \subset \ldots \subset \mathcal{E}_{n}$ the list of integers

$$
\sigma_{1} \leq \ldots \leq \sigma_{n}, \quad \sigma_{k}=\operatorname{dim} \frac{\mathcal{E}_{k}}{\mathcal{E}_{k-1}}
$$

gives the structure at infinity of $\Sigma$. 
The information contained in the structure at infinity is known to play a crucial role in the solution of many control problems ([14], [22]). Here, we recall that the following equalities hold:

$$
\sigma_{1}=\rho_{1}, \quad \sigma_{i}-\sigma_{i-1}=\rho_{i} \quad \text { for } i=1,2, \ldots
$$

where $\rho_{i}$ 's is the index obtained from the $i$ th step of the structure algorithm ([27], [15]) applied to the system $\Sigma$, in particular $\sigma_{n}=\rho$, the rank of the system.

3. Canonical form under state space transformations and feedback. In dealing with nonlinear systems of the form (2), one is interested in considering a class of transformations larger than the one used in the linear case. Adopting a differential geometric point of view as in ([14] and [22]), diffeomorphisms and (nonlinear) static state feedbacks provide a natural choice in many interesting situations. In the differential algebraic approach ([5], [6]) a more general class of transformations, involving not only the state and the output, but also a finite number of its derivatives, has proved to be appropriate.

For this class of transformation it is possible to parallel, up to some extent, the procedure that in the linear case leads to the Morse canonical form. In order to describe this, let us introduce the notions of generalized state-space transformation and of regular generalized state feedback.

DEFINITION 2. A generalized state-space transformation is a map

$$
T:\left(x, u, \dot{u}, \ldots, u^{(k)}, \ldots\right) \rightarrow\left(\xi, u, \dot{u}, \ldots, u^{(k)}, \ldots\right)
$$

such that for some $k$ we have

$$
\begin{aligned}
& \operatorname{span}\{d \xi\} \subseteq \operatorname{span}\left\{d x, d u, d \dot{u}, \ldots, d u^{(k)}\right\}, \\
& \operatorname{span}\{d x\} \subseteq \operatorname{span}\left\{d \xi, d u, d \dot{u}, \ldots, d u^{(k)}\right\} .
\end{aligned}
$$

Letting $\mathcal{X}=\operatorname{span}\{d x\}$ and $\mathcal{U}=\operatorname{span}\left\{d u, d \dot{u}, \ldots, d u^{(k)}, \ldots\right\}$, a generalized state-space transformation gives rise to an isomorphism $\tau: \mathcal{E}=\mathcal{X} \oplus \mathcal{U} \rightarrow \mathcal{E}=$ $\mathcal{X} \oplus \mathcal{U}$ satisfying the conditions

(i) $\tau(\mathcal{X}) \oplus \mathcal{U}=\mathcal{X} \oplus \mathcal{U}$

(ii) $\tau(\mathcal{X})$ is a closed subspace of $\mathcal{E}$.

In particular, this means that there exist $n$ elements $\xi_{1}, \xi_{2}, \ldots, \xi_{n} \in K$ such that $\tau(\mathcal{X})=\operatorname{span}\left\{d \xi_{1}, d \xi_{2}, \ldots, d \xi_{n}\right\}$ and $\partial\left(\xi_{1}, \xi_{2}, \ldots, \xi_{n}\right) / \partial\left(x_{1}, x_{2}, \ldots, x_{n}\right)$ is generically nonsingular.

DEFINITION 3. A regular generalized state feedback is a map

$$
F:\left(x, u, \dot{u}, \ldots, u^{(k)}, \ldots\right) \rightarrow\left(x, v, \dot{v}, \ldots, v^{(k)}, \ldots\right)
$$

such that for some $k$ we have

$$
\begin{aligned}
& \operatorname{span}\{d v\} \subseteq \operatorname{span}\left\{d x, d u, d \dot{u}, \ldots, d u^{(k)}\right\}, \\
& \operatorname{span}\{d u\} \subseteq \operatorname{span}\left\{d x, d v, d \dot{v}, \ldots, d v^{(k)}\right\} .
\end{aligned}
$$


A regular generalized state feedback gives rise to an isomorphism

$$
\varphi: \mathcal{E}=\mathcal{X} \oplus \mathcal{U} \rightarrow \mathcal{E}=\mathcal{X} \oplus \mathcal{U}
$$

such that

(i) $\mathcal{X} \oplus \varphi(\mathcal{U})=\mathcal{X} \oplus \mathcal{U}$,

(ii) $\varphi(\mathcal{U})$ is a closed subspace of $\mathcal{E}$.

This implies, in particular, that $\partial\left(v_{1}, v_{2}, \ldots, v_{m}\right) / \partial\left(u_{1}, u_{2}, \ldots, u_{m}\right)$ is generically nonsingular.

The generalized transformations we have introduced can be seen as a particular case of quasi-static feedback in the sense of [2].

Locally, this definition yields:

$$
\begin{array}{ll}
u_{i}=\phi_{i}\left(x, v, \ldots, v^{\left(\alpha_{i}\right)}\right), & i=1, \ldots, m, \\
v_{i}=\psi_{i}\left(x, u, \ldots, u^{\left(\beta_{i}\right)}\right), & i=1, \ldots, m,
\end{array}
$$

and in the rest of the paper, we will consider only the regular situation in which

$$
\operatorname{rank}(\partial \phi / \partial v)=\operatorname{rank}(\partial \psi / \partial u)=\operatorname{dim} u=\operatorname{dim} v .
$$

Concerning output injection, it could be reasonable, at this point, in order to generalize the notion used in the linear framework, to start by considering additive assignments of the form $\left.\dot{x} \mapsto \dot{x}+f\left(y, \ldots, y^{(\gamma}\right)\right)$, involving nonlinear functions of $y$ and their derivatives. Actually, the transformations of this class may have undesired properties, since they could change the observability of the system, and the introduction of some restrictive conditions is therefore required. However, we will not undertake now the task of developing an appropriate generalization of the notion of output injection, since for carrying on, up to some extent, a construction similar to that of the Morse canonical form we do not need to allow, for a given system, all possible transformations induced by assignments of the form $\dot{x} \mapsto \dot{x}+f\left(y, \ldots, y^{(\gamma)}\right)$. It will be sufficient, in fact, to use only very particular output injections of the above kind for proving the following result of [23]. A deeper discussion about the generalization of the notion of output injection will be the object of Section 5 .

Proposition 1. Given a nonlinear system of the form (2), such that generically $\operatorname{rank} g(x)=m$ and $\operatorname{rank} \partial h(x) / \partial x=p$, there exist a generalized state space transformation, a generalized state feedback and an output injection that transform the system into the following form

$$
\Sigma^{\prime}=\left\{\begin{array}{l}
\dot{\zeta}=A \zeta+B v \\
\dot{\hat{\zeta}}=f\left(\zeta, \hat{\zeta}, v, \ldots, v^{(\nu)}\right) \\
y=C \zeta
\end{array}\right.
$$

Remark that $\Sigma^{\prime}$ is linear from an input/output point of view and that the new input $v$ is obtained in terms of $x, u$ and of a finite number of derivatives of $u$. 
Proof. The proof of the statement is constructive and goes as follows. We start by constructing a suitable set of variables $(\zeta, v)$ by means of the following procedure:

St ep 0: Define $\zeta_{0}:=y$

S tep $k$ : Choose a subset of $\left(\zeta_{k}, v_{k}\right)$ of the components of $\dot{\zeta}_{k-1}$ such that

(i) $\operatorname{span}\left\{d x, d \dot{y}, \ldots, d y^{(k)}\right\}$

$$
=\operatorname{span}\left\{d x, d v_{1}, \ldots, d v_{1}^{(k-1)}, d v_{2}, \ldots, d v_{2}^{(k-2)}, \ldots, d v_{k}\right\}
$$

(ii) $\left\{d \zeta_{0}, d \zeta_{1}, d v_{1}, \ldots, d v_{1}^{(k-1)}, d \zeta_{k}, d v_{k}\right\}$ is a basis for $\operatorname{span}\left\{d x, d \dot{y}, \ldots, d y^{(k)}\right\}$.

The procedure stops when $\left(\zeta_{k+1}, v_{k+1}\right)$ is empty, namely when

- $\operatorname{span}\left\{d x, d \dot{y}, \ldots, d y^{(k+1)}\right\}=\operatorname{span}\left\{d x, d v_{1}, \ldots, d v_{1}^{(k)}, \ldots, d v_{k}, d \dot{v}_{k}\right\}$ and

- $\left\{d \zeta_{0}, d \zeta_{1}, d v_{1}, \ldots, d v_{1}^{(k)}, d \zeta_{k}, d v_{k}, d \dot{v}_{k}\right\}$ is a basis for $\operatorname{span}\left\{d x, d \dot{y}, \ldots, d y^{(k+1)}\right\}$.

A practical way of implementing the procedure described above consists essentially in applying the structure algorithm to the system. Namely, at Step 1, write

where

$$
\dot{\zeta}_{0}=\dot{y}=(\partial h / \partial x)(f(x)+g(x) u)=\left[\begin{array}{l}
y_{11}(x, u) \\
y_{12}(x, u) \\
y_{13}(x, u)
\end{array}\right]
$$

- $\partial y_{11}(x, u) / \partial u$ is full row rank and $\operatorname{rank} \partial y_{11}(x, u) / \partial u=\operatorname{rank} \partial \dot{y} / \partial u$,

- $\partial\left(y, y_{12}\right) / \partial x=$ is full row rank and $\operatorname{rank} \partial\left(y, y_{12}\right) / \partial x=\operatorname{rank} \partial\left(y, y_{12}, y_{13}\right) / \partial x$.

Then, define

$$
\begin{aligned}
& v_{1}:=y_{11}(x, u), \\
& \zeta_{1}:=y_{12}(x, u) .
\end{aligned}
$$

It is easy to check that $\left(\zeta_{1}, v_{1}\right)$ verify conditions (i), (ii) above.

At Step $k$, after reordering if necessary, write

$$
\begin{aligned}
\dot{\zeta}_{k-1} & =\dot{y}_{(k-1) 2}\left(x, u, v_{1}, \ldots, v_{1}^{(k-1)}, v_{2}, \ldots, v_{2}^{(k-2)}, \ldots, v_{k-1}, \dot{v}_{k-1}\right) \\
& =\left[\begin{array}{l}
y_{k 1}\left(x, u, \ldots, \dot{v}_{k-1}\right) \\
y_{k 2}\left(x, u, \ldots, \dot{v}_{k-1}\right) \\
y_{k 3}\left(x, u, \ldots, \dot{v}_{k-1}\right)
\end{array}\right]
\end{aligned}
$$

where

- $\partial y_{k 1} / \partial u$ is full row rank and $\operatorname{rank} \partial\left(y_{11}, \ldots, y_{(k-1) 1}, y_{k 1}\right) / \partial u=\operatorname{rank} \partial\left(y_{11}, \ldots, y_{(k-1) 1}, \dot{y}_{(k-1) 2}\right) / \partial u$,

- $\partial y_{k 2} / \partial x$ is full row rank and $\operatorname{rank} \partial\left(y, y_{12}, \ldots, y_{k 2}\right) / \partial x=\operatorname{rank} \partial\left(y, y_{12}, \ldots, y_{k 2}, y_{k 3}\right) / \partial x$. 
Then, define

$$
\begin{aligned}
v_{k} & :=y_{k 1}\left(x, u, \ldots, \dot{v}_{k-1}\right), \\
\zeta_{k} & :=y_{k 2}\left(x, u, \ldots, \dot{v}_{k-1}\right) .
\end{aligned}
$$

It is easy to check that $\left(\zeta_{k}, v_{k}\right)$ satisfy conditions (i), (ii) above.

The set of variables $\left(\zeta_{0}, \ldots, \zeta_{\nu}, v_{1}, \ldots, v_{\nu}\right)$ obtained when the procedure stops can be completed by adding block variables

$$
\begin{aligned}
v_{\nu+1} & =v_{\nu+1}(u), \\
\zeta_{\nu+1} & :=\zeta_{\nu+1}(x),
\end{aligned}
$$

in such a way that the transformation

$$
\left(\zeta_{0}, \ldots, \zeta_{\nu+1}, v_{1}, \ldots, v_{1}^{(\nu)}, \ldots, v_{\nu}, \dot{v}_{\nu}, v_{\nu+1}\right)=\phi\left(x, u, \ldots, u^{(\nu)}\right)
$$

defined by the equations (4)-(9) is a diffeomorphism, linear in $u$, on some open subset of $\Re^{(n+m(\nu+1))}$. Solving for $u$, it yields a regular generalized state feedback described locally by

$$
u=\alpha\left(x, v, \ldots, v^{k}\right)
$$

that satisfies the conditions

$$
\begin{aligned}
& \operatorname{span}\{d v\} \subseteq \operatorname{span}\left\{d x, d u, d \dot{u}, \ldots, d u^{(k)}\right\}, \\
& \operatorname{span}\{d u\} \subseteq \operatorname{span}\left\{d x, d v, d \dot{v}, \ldots, d v^{(k)}\right\} .
\end{aligned}
$$

In order to display the effect of the transformations (10) and (11) on the original system, let us remark that, when the procedure stops after $\nu$ steps (that is when $y_{(\nu+1) 1}$ and $y_{(\nu+1) 2}$ are empty), one has a partition of the vector $\zeta_{0}$, or equivalently of $y$, into $2 \nu+1$ blocks $\zeta_{0 h}$, for $1 \leq h \leq 2 \nu+1$, whose dimensions correspond, for $1 \leq h \leq \nu$, to those of the blocks $v_{h}$, or equivalently $y_{h 1}$, and, for $\nu+1 \leq h \leq 2 \nu+1$, to those of the blocks $y_{(2 \nu+2-h) 3}$ respectively (note, in fact, that we have from the above procedure $\nu$ blocks $y_{k 1}$ and $\nu+1$ blocks $y_{k 3}$, some of which may obviously be empty). Then, expressing the system in the new variables, we get

$$
\Sigma^{\prime}=\left\{\begin{array}{l}
\dot{\zeta}_{0 h}=\zeta_{1 h}, \\
\vdots \\
\dot{\zeta}_{n_{h} h}=v_{h}, \\
y_{h}=\zeta_{0 h} \quad \text { for } 1 \leq h \leq \nu, \\
\dot{\zeta}_{0 h}=\zeta_{1 h}, \\
\vdots \\
\dot{\zeta}_{n_{h} h}=f_{h}\left(\zeta_{0}, \ldots, \zeta_{\nu}\right), \\
y_{h}=\zeta_{0 h} \text { for } \nu+1 \leq h \leq 2 \nu+1, \\
\dot{\zeta}_{\nu+1}=\hat{f}\left(\zeta, v, \ldots, v^{(\nu)}\right),
\end{array}\right.
$$


Now, recalling how the variables $\zeta_{0}, \ldots, \zeta_{\nu}$ have been defined in (5), (7) the relations $\dot{\zeta}_{n_{h} h}=f_{h}\left(\zeta_{0}, \ldots, \zeta_{\nu}\right)$ can easily be modified by the particular output injection defined by the assignment

$$
\dot{\zeta}_{n_{h} h} \mapsto \dot{\zeta}_{n_{h} h}-f_{h}\left(y_{i}^{(j)}\right)
$$

with $1 \leq i \leq \nu, 1 \leq j \leq n_{h}$ and $\nu+1 \leq h \leq 2 \nu+1$. In this way we have

$$
\Sigma^{\prime \prime}=\left\{\begin{array}{l}
\dot{\zeta}_{0 h}=\zeta_{1 h}, \\
\vdots \\
\dot{\zeta}_{n_{h} h}=v_{h}, \\
y_{h}=\zeta_{0 h} \quad \text { for } 1 \leq h \leq \nu \\
\dot{\zeta}_{0 h}=\zeta_{1 h}, \\
\vdots \\
\dot{\zeta}_{n_{h} h}=0, \\
y_{h}=\zeta_{0 h} \quad \text { for } \nu+1 \leq h \leq 2 \nu+1 \\
\dot{\zeta}_{\nu+1}=\hat{f}\left(\zeta, v, \ldots, v^{(\nu)}\right)
\end{array}\right.
$$

This, denoting $\left(\zeta_{0}, \ldots, \zeta_{\nu}, \zeta_{\nu+1}\right)$ by $(\zeta, \hat{\zeta})$, gives finally the desired form

$$
\left\{\begin{array}{l}
\dot{\zeta}=A \zeta+B v \\
\dot{\hat{\zeta}}=f\left(\zeta, \hat{\zeta}, v, \ldots, v^{(\nu)}\right) \\
y=C \zeta
\end{array}\right.
$$

We can now remark the following facts about the representation (12). The subsystems described by the first two blocks of (12), that represent the observable part of $\Sigma^{\prime \prime}$, are invariant with respect to generalized state-space transformations and regular generalized state feedbacks. The first one contains the information about the algebraic structure at infinity of $\Sigma$, which corresponds to that contained in the list $I_{1}$ of the Morse canonical form. Namely, for each $h$, $1 \leq h \leq \nu$, there are as many zeros at infinity of order $n_{h}+1$ as $\operatorname{dim} \zeta_{0 h}$. In particular, with the notations of Section 2.2, we have $n_{h}+1=\sigma_{h}-\sigma_{h-1}$. The list $\left\{n_{\nu+1}, \ldots, n_{2 \nu+1}\right\}$ obtained by the second block coincides, if $\Sigma$ is linear, with the Morse list $I_{3}$.

In order to decouple the last block, as done in the linear case, we should now make use of some sort of generalized output injection. Actually, as said previously, while the generalization of the notion of feedback we employed came in a quite natural way, the situation is much more involved for output injection. We will come back to this point in a next section, after discussing some examples. 


\section{Some examples}

4.1. Example. The following system has been considered in [13]:

$$
\Sigma=\left\{\begin{array}{l}
\dot{x}_{1}=u_{1}, \\
\dot{x}_{2}=x_{4}+u_{2}, \\
\dot{x}_{3}=x_{3} u_{1}+u_{2}, \\
\dot{x}_{4}=u_{3}, \\
y_{1}=x_{1}, \\
y_{2}=x_{2}-x_{3} .
\end{array}\right.
$$

Applying the procedure described in Section 3, we obtain, at the various steps:

$$
\begin{array}{ll}
\text { Step } 0: & \zeta_{01}:=x_{1}, \\
& \zeta_{02}:=x_{2}-x_{3} . \\
\text { Step } 1: & \dot{y}=\left(\begin{array}{c}
u_{1} \\
x_{4}-x_{3} u_{1}
\end{array}\right), \quad v_{1}:=u_{1}, \\
\text { Step 2: } & \quad \ddot{y}=\left(\begin{array}{c}
\zeta_{12}:=x_{4}-x_{3} u_{1} . \\
u_{3}-x_{3} \dot{u}_{1}-x_{3} u_{1}^{2}-u_{2} u_{1}
\end{array}\right), \quad v_{2}:=u_{3}-x_{3} \dot{u}_{1}-x_{3} u_{1}^{2}-u_{2} u_{1} .
\end{array}
$$

The procedure stops, and we can complete the transformation by chosing $\zeta_{3}:=x_{4}$ and $v_{3}:=u_{3}$ so that $\left(\zeta, v_{1}, v_{2}, v_{3}, \dot{v}_{1}\right)=\phi\left(x, u_{1}, u_{2}, u_{3}, \dot{u}_{1}\right)$ is a diffeomorphism.

As a result, the system takes the form

$$
\left\{\begin{array}{l}
\dot{\zeta_{01}}=v_{1}, \\
\dot{\zeta}_{02}=\zeta_{12}, \\
\dot{\zeta}_{12}=v_{2}, \\
\dot{\zeta}_{3}=v_{3}, \\
y_{1}=\zeta_{01}, \\
y_{2}=\zeta_{02} .
\end{array}\right.
$$

4.2. Example. Let us consider the following system:

$$
\Sigma=\left\{\begin{array}{l}
\dot{x}_{1}=u_{1}, \\
\dot{x}_{2}=x_{4}+u_{2}, \\
\dot{x}_{3}=u_{2}, \\
\dot{x}_{4}=x_{3}+u_{2}, \\
y_{1}=x_{1}, \\
y_{2}=x_{2}-x_{3} .
\end{array}\right.
$$

The system is decoupable by a regular static state feedback. Then, the transformation we get by applying the procedure described in Section 3 reduces to a usual state space transformation and a regular state feedback.

At the various steps we obtain what follows:

$$
\begin{array}{ll}
\text { Step } 0: & \zeta_{01}=x_{1}, \\
& \zeta_{02}=x_{2}-x_{3} .
\end{array}
$$




$$
\begin{array}{ll}
\text { Step 1: } & \dot{y}_{1}=u_{1}=y_{11}(x, u)=v_{1}, \\
& \dot{y}_{2}=x_{4}=y_{12}(x, u)=\zeta_{12} . \\
\text { Step 2: } & \ddot{y}_{1}=\dot{u}_{1}, \\
& \ddot{y}_{2}=x_{3}+u_{2}=v_{2} .
\end{array}
$$

The procedure stops, and we can complete the transformation by defining $\zeta_{3}:=x_{3}$.

The canonical representation now takes the form

$$
\left\{\begin{array}{l}
\dot{\zeta}_{01}=v_{1}, \\
\dot{\zeta}_{02}=\zeta_{12}, \\
\dot{\zeta}_{12}=v_{2}, \\
\dot{\zeta}_{3}=v_{2} / \zeta_{3}, \\
y_{1}=\zeta_{01}, \\
y_{2}=\zeta_{02} .
\end{array}\right.
$$

5. Generalizing the notion of output injection. In order to obtain a complete analogy with the Morse canonical form in the nonlinear setting we are working in, we would now decouple $\zeta_{\nu+1}$ in (12) from $\left(\zeta_{0}, \ldots, \zeta_{\nu}\right)$ and $\left(v_{1}, \ldots, v_{\nu}\right)$ and then, possibly, we would split the corresponding block into a controllable and a noncontrollable part. In decoupling, we would use only transformations that may be viewed as a generalization of the notion of output injection, followed possibly by changes of coordinates.

In the nonlinear framework, linear output injections have been used in [12]. An additive nonlinear output injection, similar to the one used in Section 3 has been employed in [18] for linearizing a nonlinear system, as well as in [9], [10] for transforming a nonlinear system into a bilinear one. In general, however, the problem of defining a quite general notion of output injection in a nonlinear framework has not received much attention in the literature.

Here, we consider a class of output injections, which are not necessarily additive and whose definition is consistent with that of quasi-static state feedback formalized in [2]. The basic idea is that of considering transformations that modify the dynamics of a system of the form (2) by the assignment

$$
\dot{x} \longrightarrow \theta\left(\dot{x}, y, \dot{y}, \ldots, y^{(r)}\right) .
$$

In other terms, this amounts to transform the system

$$
\Sigma=\left\{\begin{array}{l}
\dot{x}=f(x)+g(x) u, \\
y=h(x),
\end{array}\right.
$$

into

$$
\widetilde{\Sigma}=\left\{\begin{array}{l}
\dot{x}=\theta\left(f(x)+g(x) u, y, \dot{y}, \ldots, y^{(r)}\right), \quad r \in N, \\
y=h(x) .
\end{array}\right.
$$

In the following, in order to make distinction between the derivatives of $y$ along the trajectories of (14) and those along the trajectories of (15), we will denote 
the latter ones by $y^{[k]}$, whereas the notation $y^{(k)}$ will stand for the first ones.

Clearly, some restrictions must limit the choice of the function $\theta(f(x)+$ $\left.g(x) u, y, \dot{y}, \ldots, y^{(r)}\right)$, in order to avoid pathological situations. In particular, we would prevent the possibility that an output injection can change the observability properties of the system. Let us illustrate this point by considering the following example. Let us apply to the system

$$
\left\{\begin{array}{l}
\dot{x}_{1}=0, \\
\dot{x}_{2}=0, \\
y=x_{1} x_{2},
\end{array}\right.
$$

which is obviously not fully observable, the linear, additive output injection defined by $\theta(\dot{x}, y)=\left(\dot{x}_{1}+y, 0\right)^{t}$. The resulting system is:

$$
\left\{\begin{array}{l}
\dot{x}_{1}=x_{1} x_{2}, \\
\dot{x}_{2}=0, \\
y=x_{1} x_{2},
\end{array}\right.
$$

which turns out to be completely observable (in the sense of [4]) since $x_{1}=y^{2} / y^{[1]}$ and $x_{2}=y^{[1]} / y$.

Before going further, it is therefore useful to state formally, following [29], the notion of observability we will consider in defining the notion of output injection (see also [11]).

Definition 4. Given a system of the form (14), let $\mathcal{X}$ and $\mathcal{U}$ denote, as usual, $\operatorname{span}\{d x\}$ and $\operatorname{span}\left\{d u^{(k)}, k \geq 0\right\}$ respectively, and let $\mathcal{Y}$ denote $\operatorname{span}\left\{d y^{(k)}, k \geq\right.$ $0\}$. Then, the observability space of the system is defined as:

$$
\mathcal{O}=\mathcal{X} \cap(\mathcal{Y}+\mathcal{U})
$$

Definition 5. A system of the form (14) is said to be observable if and only if $\operatorname{dim}(\mathcal{X} \cap(\mathcal{Y}+\mathcal{U}))=n$, i.e. if and only if $\operatorname{span}\{d x\} \subset \operatorname{span}\left\{d y^{(h)}, d u^{(k)}, h \geq\right.$ $0, k \geq 0\}$.

Now, given a system of the form (14) and a map of the form (13), we can consider the vector spaces $\mathcal{H}_{k}=\operatorname{span}\left\{d y, d \dot{y}, \ldots, d y{ }^{(k)}, d u, d \dot{u}, \ldots\right\}$ and $\widetilde{\mathcal{H}}_{k}=$ $\operatorname{span}\left\{d y, d y^{[1]}, \ldots, d y^{[k]}, d u, d \dot{u}, \ldots\right\}$. With these notations it is possible to state, as in [20], the following definition of output injection (see also [1]).

Definition 6. The map $\theta\left(\dot{x}, y, \dot{y}, \ldots, y^{(r)}\right)(r \in N)$ that transforms the system (14) into the system (15) is an output injection if the following conditions hold:

- $\widetilde{H}_{k} \subset H_{k+r-1}$,

- $H_{k} \subset \tilde{H}_{k+r-1}$,

- $\partial \theta\left(\dot{x}, y, \dot{y}, \ldots, y^{(r)}\right) / \partial \dot{x}$ is generically invertible.

The following Proposition, proved in [20], states that the output injection defined above behaves well with respect to the observability.

Proposition 2. Given a system of the form (14) and a map $\theta$ of the form (13), let $\mathcal{O}$ denote the observability space of (14) and let $\widetilde{\mathcal{O}}=\mathcal{X} \cap(\widetilde{\mathcal{Y}}+\mathcal{U})$, where 
$\widetilde{\mathcal{Y}}=\operatorname{span}\left\{d y^{[k]}, k \geq 0\right\}$, denote the observability space of (15). If $\theta$ is an output injection, then $\mathcal{O}=\widetilde{\mathcal{O}}$.

Let us now go back to the system $\Sigma^{\prime \prime}$ described by (12) at the end of Section 3. Denoting the block variables $\left(\zeta_{0 h}, \ldots, \zeta_{n_{h} h}\right)$ for $0 \leq h \leq \nu$, that is the variables of the first block in (12), by $\zeta_{1}$, the block variables $\left(\zeta_{0 h}, \ldots, \zeta_{n_{h} h}\right)$ for $\nu+1 \leq$ $h \leq 2 \nu+1$, that is the variables of the second block in (12), by $\zeta_{2}$ and the block variable $\zeta_{\nu+1}$ by $\zeta_{3}$, and analogously denoting the block variables $\left(v_{1}, \ldots, v_{\nu}\right)$ by $w_{1}$ and $v_{\nu+1}$ by $w_{2}$, we can rewrite (12) as

$$
\left\{\begin{array}{l}
\dot{\zeta}_{1}=f_{1}\left(\zeta_{1}, w_{1}\right), \\
\dot{\zeta}_{2}=f_{2}\left(\zeta_{2}\right), \\
\dot{\zeta}_{3}=f_{3}\left(\zeta_{1}, \zeta_{2}, \zeta_{3}, w_{1}, \ldots, w_{1}^{(\mu)}, w_{2}\right), \\
y=h\left(\zeta_{1}, \zeta_{2}\right) .
\end{array}\right.
$$

Let us focus on the subsystem

$$
\left.\dot{\zeta}_{3}=f_{3}\left(\zeta_{1}, \zeta_{2}, \zeta_{3}, w_{1}, \ldots, w_{1}^{(\mu)}, w_{2}\right)\right)
$$

corresponding to the unobservable variable $\zeta_{3}$. The problem of decoupling $\zeta_{3}$ from $\zeta_{1}, \zeta_{2}$ and $w_{1}$ by means of an output injection does not seem to be tractable in general. So, we limit our attention to the situations in which $f_{3}$ has particular properties. Namely, we assume that, for each component $f_{3 i}$ of $f_{3}$, there exists a function $\chi_{i}\left(\zeta_{3}, w_{2}\right)$ such that:

$$
f_{3 i}=F_{i}\left(\chi_{i}\left(\zeta_{3}, w_{2}\right), \zeta_{1}, \zeta_{2}, w_{1}, \ldots, w_{1}^{(\mu)}\right) .
$$

In other terms, we assume that there exists a separation function of the observable variables and the unobservable ones.

Then, we have the following two cases: either $f_{3 i}$ does not depend on $\zeta_{3}$ and on $w_{2}$, and we chose $\chi_{i}\left(\zeta_{3}, w_{2}\right)=0$, or $\left(\partial F_{i} / \partial \chi_{i}\right) \neq 0$. Now, by the construction carried on in the proof of Proposition $1, \zeta_{1}, \zeta_{2}$ and $w_{1}^{(k)}$ can be expressed in terms of $y$ and its derivatives. Hence we can define, in the case in which $f_{3 i}$ does not depend on $\zeta_{3}$ and on $v_{2}$,

$$
\theta_{i}\left(\dot{\zeta}_{3 i}, y, \dot{y}, \ldots, y^{(r)}\right)=\dot{\zeta_{3 i}}-F_{i}\left(\zeta_{1}, \zeta_{2}, w_{1}, \ldots, w_{1}^{(\mu)}\right) .
$$

In the case in which $\partial F_{i} / \partial \chi_{i} \neq 0$, we can apply the implicit function theorem to get

$$
\chi_{i}=G_{i}\left(f_{3 i}, \zeta_{1}, \zeta_{2}, w_{1}, \ldots, w_{1}^{(\mu)}\right)
$$

and we define

$$
\theta_{i}\left(\dot{\zeta_{3 i}}, y, \dot{y}, \ldots, y^{(r)}\right)=G_{i}\left(\dot{\zeta}_{3 i}, \zeta_{1}, \zeta_{2}, w_{1}, \ldots, w_{1}^{(\mu)}\right) .
$$

The map $\theta=\left(\theta_{1}, \ldots, \theta_{i}, \ldots\right)$ defines an output injection according to Definition 6 which transforms (16) into

$$
\dot{\zeta}_{3}=\chi\left(\zeta_{3}, w_{2}\right)
$$


where $\chi=\left(\chi_{1}, \ldots, \chi_{i}, \ldots\right)$, achieving the desired decoupling and yielding a maximal loss of accessibility.

Obviously, the separability condition seen above is only sufficient for assuring the possibility of decoupling $\zeta_{3}$ by means of an output injection. In addition, the fact that such condition is not feedback invariant, as pointed out in [25], shows that a complete characterization of existence of an output injection with the desired property is still far from being obtained.

\section{Examples}

6.1. Example. Let us consider the following system:

$$
\left\{\begin{array}{l}
\dot{x}_{1}=v_{1}, \\
\dot{x}_{2}=x_{2}^{2} x_{1}+x_{1} v_{2}+x_{1}^{2}, \\
y=x_{1} .
\end{array}\right.
$$

For the nonobservable part, we can write $\dot{x}_{2}=\left(x_{2}^{2}+v_{2}\right) x_{1}+x_{1}^{2}$. Hence, choosing $\chi\left(x_{2}, v_{2}\right)=\left(x_{2}^{2}+v_{2}\right)$, we have $\dot{x}_{2}=F\left(\chi, x_{1}\right)$ and $\chi=\left(\dot{x}_{2}-x_{1}^{2}\right) / x_{1}$. Remarking that $x_{1}=y$, we can define the output injection

$$
\theta\left(\dot{x_{2}}, y\right)=\left(\dot{x}_{2}-x_{1}^{2}\right) / x_{1}
$$

Finally, we obtain the system

$$
\left\{\begin{array}{l}
\dot{x}_{1}=v_{1} \\
\dot{x}_{2}=x_{2}^{2}+v_{2} \\
y=x_{1}
\end{array}\right.
$$

6.2. Example. Let us consider the following system:

$$
\left\{\begin{array}{l}
\dot{x}_{1}=v_{1}, \\
\dot{x}_{2}=x_{2}^{2} x_{1}+v_{2}, \\
y=x_{1} .
\end{array}\right.
$$

Here, it is not possible to write, for the right hand side of the nonobservable block, $f_{2}=F\left(\chi\left(x_{2}, v_{2}\right), x_{1}, v_{1}\right)$. In fact, assuming that this holds for some $F$ and $\chi$, one has

(i) $\frac{\partial f_{2}}{\partial v_{2}}=1=\frac{\partial F}{\partial \chi} \frac{\partial \chi}{\partial v_{2}}$,

(ii) $\frac{\partial}{\partial x_{1}}\left(\frac{\partial f_{2}}{\partial v_{2}}\right)=0=\left(\frac{\partial}{\partial x_{1}}\left(\frac{\partial F}{\partial \chi}\right)\right) \frac{\partial \chi}{\partial v_{2}}$,

(iii) $\frac{\partial}{\partial x_{1}}\left(\frac{\partial f_{2}}{\partial x_{2}}\right)=2 x_{2}=\left(\frac{\partial}{\partial x_{1}}\left(\frac{\partial F}{\partial \chi}\right)\right) \frac{\partial \chi}{\partial x_{2}}$.

Now (i) implies $\partial \chi / \partial v_{2} \neq 0$, while (ii) and (iii) imply $\partial \chi / \partial v_{2}=0$, that is a contradiction.

As a consequence, there is no separation function in this case and, actually, the nonobservable block cannot be decoupled from $x_{1}$.

Acknowledgments. The authors wish to thank the unknown referees and W. Respondek for valuable comments and suggestions. 


\section{References}

[1] G. Conte, C. H. Moog, A. M. Perdon and Y. F. Zheng, A generalized state space decomposition of nonlinear systems, in: Proc. 31st CDC IEEE, Tucson, 1992, 3676-3677.

[2] E. Delaleau and M. Fliess, An algebraic interpration of the structure algorithm with an application to feedback decoupling, in: Nonlinear Control Systems Design IFAC Symposium, Bordeaux, 1992, 489-494.

[3] M. D. Di Benedetto, J. W. Grizzle and C. H. Moog, Rank invariants for nonlinear systems, SIAM J. Control Optim. 27 (1989), 658-672.

[4] S. Diop and M. Fliess, On nonlinear observability, in: European Control Conference Grenoble, 1991, 152-157

[5] M. Fliess, Nonlinear control theory and differential algebra, in: Proc. I.I.A.S.A. Conference on Modelling and Adaptive Control, Sopron, Hungary, 1986.

[6] - Automatique et corps différentiels, Forum Math. 1 (1989), 227-238.

[7] —, Généralisation nonlinéaire de la forme canonique de commande et linéarisation par bouclage, C. R. Acad. Sci. Paris 308 (1989), 377-379.

[8] - Generalized controller canonical forms for linear and nonlinear dynamics, I.E.E.E. Trans. Automat. Control 35 (1990), 994-1001.

[9] H. Hammouri and J. P. Gauthier, Bilinearization up to output injection, Systems and Control Lett. 11 (1988), 139-149.

[10] - - - Global time-varying linearization up to output injection, SIAM J. Control Optim. 30 (1992), 1295-1310.

[11] R. Hermann and A. J. Krener, Nonlinear controllability and observability, IEEE Trans. Automat Control AC-22 (1977), 728-740.

[12] A. Isidori, Nonlinear feedback, structure at infinity and the input-output linearization problem, in: Proc. MTNS 83, Beer Sheva, Lecture Notes in Control and Inform. Sci. 58, Springer, Berlin, 1984, 473-493.

[13] - Control of nonlinear systems via dynamic state-feedback, in: Algebraic and Geometric Methods in Nonlinear Control Theory, M. Fliess and M. Hazewinkel (eds.), Reidel, Dordrecht, 1986, 121-145.

[14] —, Nonlinear Control Systems, 2nd ed., Springer, Berlin, 1989.

[15] A. Isidori and C. H. Moog, On the equivalence of the notion of transmission zeros, in: Modelling and Adaptive Contro, Proc. IIASA Conf., Sopron, Hungary, C. I. Byrnes and A. Kurszanski (eds.), Lecture Notes Control and Inform. Sci. 105, Springer, 1988, Berlin, $146-158$.

[16] T. Kailath, Linear Systems, Prentice-Hall, New York, 1980

[17] A. J. Krener, Normal form for linear and nonlinear sytems, in: Contemp. Math. 68, Amer. Math. Soc., 1987, 157-189.

[18] A. J. Krener and A. Isidori, Linearization by output injection and nonlinear observers, Systems Control Lett. 3 (1983), 47-52.

[19] R. Marino, W. Respondek and A. J. van der Shaft, Equivalence of nonlinear systems to input-output prime forms, SIAM J. Control Optim., to appear.

[20] C. H. Moog, F. Plestan, G. Conte and A. M. Perdon, On canonical forms of nonlinear systems, in: Proc. ECC 93, Groningen, 1993, 1514-1517.

[21] A. S. Morse, Structural invariants of linear multivariable systems, SIAM J. Control Optim. 11 (1973), 446-465

[22] H. Nijmeijer and A. J. van der Shaft, Nonlinear Dynamics Control Systems, Springer, 1990

[23] A. M. Perdon, G. Conte and C. H. Moog, Some canonical properties of nonlinear systems, in: Realization and Modelling in System Theory, Proc. MTNS 89, Amsterdam, 1989, 89-96. 
[24] A. M. Perdon, Y. F. Zheng, C. H. Moog and G. Conte, Disturbance decoupling for nonlinear systems: a unified approach, Kybernetica 29 (1993), 479-484.

[25] W. Respondek, personal communication, 1994.

[26] J. Rudolph, Une forme canonique en bouclage quasi-statique, C.R. Acad. Sci. Paris 316 (1993), 1323-1328.

[27] S. N. Singh, A modified algorithm for invertibility in nonlinear systems, IEEE Trans. Automat. Control AC-26 (1981), 595-598.

[28] M. Zeitz, Canonical forms for nonlinear systems, in: Geometric Theory of Nonlinear Control Systems, B. Jakubczyk, W. Respondek and K. Tchon (eds.), Wrocław, Wydawnictwo Politechniki Wrocławskiej, 1985.

[29] Y. F. Zheng and L. Cao, Transfer structure of nonlinear systems, Tech. Rep., East China Normal Univ., 1993. 